\title{
On the number of anticonformal automorphisms of a Riemann surface
}

\author{
By Ryōhei TsujI
}

(Received August 23, 1959)

Let $R$ be an orientable Riemann surface of genus $g$ with $k$ boundary components, $N$ the number of conformal mappings of $R$ onto itself and $\bar{N}$ the number of anticonformal mappings of $R$ onto itself. If $k$ is not zero, we set the following restriction; Let $R$ be a finite subsurface of another Riemann surface and its relative boundary consist of $k$ analytic curves. In this paper, we shall prove the next theorem.

THEOREM A. For any given $g$ and $k$ we have

$$
\operatorname{Max} N=\operatorname{Max} \bar{N}
$$

where maxima are taken over all orientable Riemann surfaces respectively.

We first prepare two lemmas.

Lemma 1. For each $R, \bar{N}$ is equal to either $N$ or 0 .

Proof. Let $\mathbb{S}$ be the group of all conformal mappings of $R$ onto itself and $\mathfrak{A}$ the set of all anticonformal mappings of $R$ onto itself. If $\mathfrak{A}$ is not empty, there is at least one $A \in \mathfrak{A}$. For each element $T_{i} \in \mathbb{S}$, there corresponds $A T_{i} \in \mathfrak{A}$. Conversely, for each $A_{j} \in \mathfrak{A}$, there corresponds $A^{-1} A_{j} \in \mathbb{S}$. This correspondence is surely one-to-one, so that $\bar{N}$ is equal to $N$ unless $\mathfrak{A}$ is empty.

LEMMA 2. There exists at least one compact Riemann surface of genus $g$ which has an anticonformal mapping with one fixed line.

Proof. The algebraic genus, i. e. the genus of the double of a Riemann surface with $m$ boundary components, $h$ hundles and $c$ cross-caps is given by

$$
G=2 h+m+c-1 \text {. }
$$

For $m=1$, the equation $g=2 h+c$ has always non-negative integral solutions, whatever $g$ may be. Thus the desired surface can be obtained as the double of another surface.

Next, we state two results which have been established by A. Hurwitz and K. Oikawa.

Let $R_{0}$ be a compact Riemann surface of genus $g$. We fix $k$ points $p_{1}, \cdots, p_{k}$ on $R_{0}$. Let $N_{0}$ be the number of all conformal mappings of $R_{0}-\left\{p_{1}, \cdots, p_{k}\right\}$ onto itself. For fixed $g$ and $k, \operatorname{Max} N=\operatorname{Max} N_{0}$. This has been proved by K. Oikawa [2].

Let $R$ be a compact Riemann surface of genus $g$ which has the group of conformal mappings of maximal order. Let $\mathbb{S}$ be the group of all conformal mappings of $R$ onto itself. By identifying the points congruent with respect to $\mathbb{S}$, 
we get a new surface $R_{1}$. Let $\pi$ be the genus of $R_{1}$, and $a_{1}, \cdots, a_{w}$ be the branch points on $R$.

We connect $a_{1}, \cdots, a_{w}$ with an inner point $p$ of $R_{1}$ by non-intersecting paths, and cut $R_{1}$ along these paths and homology bases of $R_{1}$. Thus, we get a simplyconnected region $R_{2}$. $R$ may be regarded as an $N$-sheeted covering of $R_{2}$ and is constructed from $N$ copies of $R_{2}$ by a suitable patching-work. The reconstruction depends only on the abstract structure of $\mathbb{B}$ as well as the multiplicities of branchpoints.

Now, we consider another simply-connected region $R_{2}{ }^{\prime}$. We take arbitrary $w$ points $a_{1}{ }^{\prime}, \cdots, a_{w}{ }^{\prime}$ on $R_{2}{ }^{\prime}$. By the same way as was used in reconstructing $R$ from $R_{2}$, we get a new compact Riemann surface $R^{\prime}$ from $R_{2}{ }^{\prime}$. The group $\mathscr{S}^{\prime}$ of all conformal mappings of $R^{\prime}$ onto itself has a subgroup which is isomorphic to $\mathbb{S}$.

Above discussion was shown in detail by A. Hurwitz [1], and this construction is essential for our present purpose. Now we proceed to prove the theorem A, by showing constructively the existence of an anticonformal mapping.

Let $R_{0}$ be a Riemann surface of genus $g$ and with $k$ boundary components which has the maximal number of conformal mappings. From Oikawa's work, we may take, instead of $R_{0}$, a compact Riemann surface $R$ of genus $g$ and with $k$ invariant points. From the conformal mapping group $\&$ of $R$, we can determine, following Hurwitz's idea stated above, $R_{1}, \pi, R_{2}, a_{1}, \cdots, a_{w}$ and their multiplicities. $k$ points $p_{1}, \cdots, p_{k}$ may lie on $a_{1}, \cdots, a_{w}$ or not. Let the projections of these points on $R_{1}$ be $q_{1}, \cdots, q_{s}$.

By lemma 2, there exists a compact Riemann surface $R_{1}{ }^{\prime}$ of genus $\pi$ which has one fixed line. Since it can be regarded as the double of another finite Riemann surface, there exists a symmetic homology basis. On the fixed line we set $w$ points $a_{1}{ }^{\prime}, \cdots, a_{w}{ }^{\prime}$ and $s$ points $q_{1}{ }^{\prime}, \cdots, q_{s}{ }^{\prime}$. These $s$ points are to be situated in the same manner as on $R_{1}$, that is, $q_{i}{ }^{\prime}$ is to be situated on $a_{j}{ }^{\prime}$ when $q_{i}$ is situated on $a_{j}$ and conversely.

We choose on the fixed line a point $p^{\prime}$ which coincides with neither $q_{i}{ }^{\prime}$ nor $a_{j}{ }^{\prime}$. We connect $p^{\prime}$ with each of $a_{j}{ }^{\prime}$ by non-intersecting paths and cut $R_{1}{ }^{\prime}$ along these and symmetric homology basis.

We thus get a new simply-connected region $R_{2}{ }^{\prime}$. We construct a new surface $R^{\prime}$ according to Hurwitz's method of reconstruction. Then, $R^{\prime}$ is a Riemann surface of genus $g$ and with $k$ fixed points, and the group $\mathbb{S}^{\prime}$ of conformal mappings of $R^{\prime}$ onto itself has a subgroup which is isomorphic to $\mathbb{G}$. But we have supposed that for a given pair of $g$ and $k, R$ has the conformal mapping group of maximal order. Hence the order of $\mathbb{B}^{\prime}$ is equal to $N$.

It remains to show the existence of an anticonformal mapping of $R^{\prime}$ onto itself. If the existence is assured, the proof of our theorem will be completed by means of Lemma 1.

We denote a point of $R^{\prime}$ by $T_{i}(p)$, where $T_{i}$ is an element of $\$$ and $p$ is a point of $R_{2}{ }^{\prime}$. Except boundary of $R_{2}{ }^{\prime}$, this notation is allowed, because $R^{\prime}$ was constructed from $R_{2}^{\prime}$ by means of the abstract structure of $\mathbb{G}$. Since $R_{1}{ }^{\prime}$ has an anticonformal mapping with one fixed line, $R_{2}{ }^{\prime}$ has also an anticonformal mapping $A_{0}$ with fixed lines. There corresponds $T_{i}(p)$ to $T_{i}{ }^{-1}\left\{A_{0}(p)\right\}$. This correspondence is an anticonformal mapping in every $T_{i}\left(R_{2}{ }^{\prime}\right)$. Concerning boundary of $R_{2}{ }^{\prime}$, $T_{j}^{-1}\left\{A_{0}\left(R_{2}^{\prime}\right)\right\}$ and $T_{i}^{-1}\left\{A_{0}\left(R_{2}^{\prime}\right)\right\}$ are adjacent whenever $T_{j}\left(R_{2}^{\prime}\right)$ and $T_{i}\left(R_{2}^{\prime}\right)$ are adjacent. Hence the above anticonformal mapping is continuable across the boun- 
dary of $R_{2}{ }^{\prime}$. Thus the existence of an anticonformal mapping of $R^{\prime}$ onto itself is assured, and our theorem has been proved.

Shibaura Institute of Technology.

\section{References}

[1] Hurwitz, A., Über algebraische Gebilde mit eindeutigen Transformationen in sich. Math. Ann. 41 (1893), 403-442.

[2] Oikawa, K., A supplement to "Note on conformpl mappings of a Riemann surfpce onto itself". Kōdai Math. Sem. Rep. 8 (1951), 115-116. 